\title{
Hospital Decision-Making Regarding the Smallpox Pre-Event Vaccination Program
}

\author{
ALEX R. KEMPER, ANNE E. COWAN, PAMELA L. Y. H. CHING, MATTHEW M. DAVIS, \\ EMILY J. KENNEDY, SARAH J. CLARK, and GARY L. FREED
}

Objectives: To understand the factors underlying the decision by U.S. hospitals to participate or not in the U.S. Smallpox Pre-Event Vaccination Program (SPVP).

Methods: We conducted semistructured telephone interviews with a convenience sample of 123 hospital decision-makers in nine states between June and November 2003.

Results: Within our sample, 88 hospitals (72\%) decided to participate in the SPVP and 35 (28\%) decided against doing so. Nearly all hospital decision-makers considered the risk of a smallpox outbreak, risks associated with vaccination, hospital costs, and the reaction of hospital stakeholders. However, these factors often were weighed differently by hospitals that decided to participate compared to those that did not. Fewer than half of all hospitals reported that public health officials played an important role in their decision-making process, but those that did felt the influence of public health officials was positive.

Conclusions: Strengthening the linkage between the public and private health sectors may help to address some of the barriers to broader participation by hospitals in the SPVP and foster the success of smallpox outbreak response preparedness efforts in the future.

B EGINNING IN JANUARY 2003, two advisory committees to the Centers for Disease Control and Prevention (CDC) - the Advisory Committee on Immunization Practices (ACIP) and the Healthcare Infection Control Practices Advisory Committee (HICPAC)—recommended that hospitals prepare teams of health-care workers for responding to smallpox outbreaks. ${ }^{1}$ The recommendations specified that within each hospital a broad range of workers - including physicians, nurses, respiratory therapists, radiology technicians, security personnel, and housekeeping staff_-be vaccinated against smallpox so that in the event of a smallpox outbreak, response teams could provide continuous care for patients with suspected or confirmed smallpox until additional healthcare workers could be vaccinated. ${ }^{1}$ State and local public health departments, with the assistance of CDC, were responsible for assisting hospitals in forming these response teams as part of the national Smallpox Pre-Event Vaccination Program (SPVP).

Therefore, initial plans for responding to a smallpox outbreak depended substantially on the willingness of individual hospitals to voluntarily participate in the SPVP, but hundreds of hospitals chose not to participate. ${ }^{2}$ While recent studies have explored the decisions of individual health-care workers regarding whether to be vaccinated against smallpox, ${ }^{3-7}$ little is known about the decisionmaking process at the institution level regarding participation in the SPVP. Understanding how hospital decision-makers determined their institution's policy regarding participation in the SPVP is important for the

Alex R. Kemper, MD, MPH, MS; Anne E. Cowan, MPH; Matthew M. Davis, MD, MAPP; Emily J. Kennedy, MPH; Sarah J. Clark, MPH; and Gary L. Freed, MD, MPH, are all with the Child Health Evaluation and Research (CHEAR) Unit, Division of General Pediatrics, University of Michigan, Ann Arbor. Pamela L. Y. H. Ching, MS, SD, RD, LD, is with the Health Services Research and Evaluation Branch, Immunization Services Division, National Immunization Program, Centers for Disease Control and Prevention, Atlanta, Georgia. 
success of future bioterrorism preparedness activities and may have implications for the delivery of public health services in the event of other large-scale outbreaks.

\section{METHODS}

\section{Hospital Selection}

The initial sampling frame was composed of 24 states that had experienced mixed success in recruiting hospitals to participate in the SPVP, with an estimated 25-75\% of the hospitals recruited agreeing to participate (P. Ching, CDC, personal communication, May 19, 2003). State smallpox preparedness coordinators in these 24 states were contacted by CDC's National Immunization Program to solicit their cooperation with this study. Nine coordinators-from California, Kentucky, Maine, Michigan, Minnesota, Mississippi, Utah, Virginia, and Wyoming - agreed to assist with the study and provided information on hospitals in the state recruited to participate in the SPVP. The number of health-care workers vaccinated in these states ranged from 63 in Maine to 1,611 in California, with a mean of $771 .^{8}$

A statewide list of hospitals recruited for the SPVP was available for six of the nine states (Kentucky, Maine, Minnesota, Utah, Virginia, Wyoming). For California, information was available for hospitals in 8 of the state's 62 local health department districts. In Michigan, hospital data were provided by 7 of the state's 8 bioterrorism planning regions. For Mississippi, the state contact forwarded to us the information for 5 hospitals that were willing to be contacted for an interview. The lists of hospitals provided by these states ranged in number from 5 hospitals in Mississippi to 108 hospitals in Kentucky, with a mean of 60 hospitals.

From these lists, we prepared our sampling frame by including only acute care hospitals. We further excluded hospitals with fewer than 25 beds, as well as children's and Veterans Administration (VA) hospitals. With a target of choosing up to 20 hospitals per state, sample hospitals were then chosen to represent a range of hospitals in the state with variation in size, county, affiliation (e.g., corporate, religious), and decision to participate in the SPVP. The state-specific samples ranged from 5 hospitals in Mississippi to 21 hospitals in Utah, with an overall sample of 148 hospitals.

\section{Interview Process}

Between June and November 2003, hospital administrators at the 148 hospitals in the sample were contacted to request a telephone interview regarding their decision to participate or not in the SPVP. Interviews were conducted with the chief executive officer (CEO) of each hospital or his or her designee who was familiar and involved with the decision-making process. Each interview took approximately 20 minutes to complete and used a standardized semistructured telephone interview format.

Respondents were first asked about the individuals involved in making the decision, the hospital's ultimate decision, and the factors that were considered during the decision-making process. Interviewees were then prompted, if they had not mentioned them, to describe how each of the following four specific categories of factors were considered in their decision-making process: (1) the risk of a smallpox outbreak; (2) the risk of vaccination, including the potential for adverse events in vaccinees, their patients, or close contacts, and the perceived need to develop policies for these vaccinated employees; (3) costs to the hospital, including compensation for adverse events, liability, and other financial or opportunity costs; and (4) stakeholder influences, including those of employees, the general public, and other outside parties (such as government agencies). Finally, after reviewing all factors considered in the decision-making process, respondents were asked to discuss the key factor or factors that influenced their decision.

This study was approved by the University of Michigan Medical Center Institutional Review Board.

\section{Data Analysis}

During each interview, a note-taker documented the critical factors influencing the decision-making process that were mentioned by interviewees. A detailed question-by-question summary, which paralleled the structure of the interview questionnaire, was then produced for each interview. Using this summary, two investigators independently coded the factors that were mentioned as influencing that institution's decision, with any differences resolved through consensus with a third investigator. The factors identified were tallied based on whether or not the hospital decided to participate in the SPVP. Representative statements from the interview summaries are included in quotation marks in the Results section below to provide context for the overall findings. To protect the privacy of the subjects, the interviews were not recorded. Therefore, these statements are paraphrased from the interview summaries. Accompanying each quotation is a symbol that denotes whether that hospital chose to participate in the SPVP $(\dagger)$ or not $(\ddagger)$.

\section{RESULTS}

Of the 148 hospitals contacted, 123 (83\%) interviews were completed. Although data on hospital size, location, and affiliation were collected, analyses to assess differ- 
ences in responses based on these variables were not performed due to the small sample size.

\section{Decision to Participate in the SPVP}

Overall, 88 of the 123 hospitals (72\%) for which interviews were completed decided to participate in the SPVP. Of these 88 hospitals, no staff members were vaccinated in $22(25 \%)$, mainly because of the lack of interested or eligible staff or the insufficient access to smallpox vaccine or vaccination clinics. Because the original intent of these 22 hospitals was to participate in the SPVP, their results have been included with the other 66 hospitals that decided to participate in the SPVP and vaccinated staff members.

\section{Factors in the Decision-Making Process}

For the four categories of factors that were specifically addressed in the interview protocol, Table 1 summarizes the proportion of hospitals that considered each factor in choosing whether to participate in the SPVP. Regardless of their decision, nearly all hospitals considered the risk of a smallpox outbreak, the risks associated with vaccination, and the costs to the hospital in making their decision, and most also considered the influence of certain stakeholders. However, these factors were often weighed differently by hospitals, both within and between the groups that did and did not decide to participate in the SPVP.
Risk of a smallpox outbreak

About $90 \%$ of all hospitals considered issues related to the likelihood of a smallpox outbreak in their decision. Many tried to assess the risk of a smallpox outbreak, taking into account the status of the war with Iraq at the time of their decision and the perceived credibility of the intelligence that an outbreak of smallpox was possible.

To focus on a more tangible risk, many decision-makers evaluated the likelihood that a smallpox case would present to their hospital in the event that an outbreak occurred. Their assessment was based on their perception of the hospital's susceptibility to exposure (e.g., proximity to metropolitan areas, likelihood of receiving a smallpox case as a transfer patient). As one respondent indicated:

\footnotetext{
"[The risk of exposure was possible] . . . due to the location of the hospital, the potential for treating military men, and the fact that the hospital is the only one in the area and has the largest emergency room in the state." $\dagger$
}

The perception of risk did not necessarily correlate with the final decision on whether to participate in the SPVP. Almost all of the hospitals interviewed felt that the risk was low, yet the majority of these hospitals decided to participate in the SPVP. The following two participating hospitals had different perceptions of risk:

"We believe there is the possibility of an outbreak and decided that doing nothing would be the greater crime." $\$$

Table 1. Factors Considered by Hospitals in Choosing Whether to Participate in the Smallpox Pre-Event Vaccination Program (SPVP)

\begin{tabular}{lcc}
\hline & $\begin{array}{c}\text { Chose to } \\
\text { participate } \\
\text { SPVP } \\
\text { Factor }\end{array}$ & $\begin{array}{c}\text { Chose not to } \\
\text { participate in } \\
\text { SPVP } \\
(\mathrm{n}=88)\end{array}$ \\
\hline Risk of a smallpox outbreak & $88 \%(77)$ & $91 \%(32)$ \\
Risks of vaccination & $90 \%(79)$ & $100 \%(35)$ \\
Potential for adverse events in vaccinees & $82 \%(72)$ & $97 \%(34)$ \\
Exposure of patients/close contacts of vaccinees & $63 \%(55)$ & $80 \%(28)$ \\
Need for policies for vaccinated employees & $57 \%(50)$ & $60 \%(21)$ \\
Costs to the hospital & $91 \%(80)$ & $94 \%(33)$ \\
Compensation for adverse events & $78 \%(69)$ & $89 \%(31)$ \\
Liability & $77 \%(68)$ & $83 \%(29)$ \\
Other financial or opportunity costs & $28 \%(25)$ & $26 \%(10)$ \\
Stakeholder influences & $72 \%(63)$ & $74 \%(26)$ \\
Employees & $38 \%(33)$ & $37 \%(13)$ \\
General public & $34 \%(30)$ & $26 \%(9)$ \\
Other outside parties & $44 \%(39)$ & $49 \%(17)$ \\
\hline
\end{tabular}


"We were not convinced of any risk of a large-scale outbreak, and small-scale outbreaks, which are more likely, have been handled effectively by ring vaccination." $\dagger$

... as did these nonparticipants:

"The hospital is located near an airport that services transatlantic flights. We often care for people who have recently come off these flights, and therefore consider ourselves somewhat at risk of treating a smallpox patient."”

"The war with Iraq was the impetus for a national program, but since no weapons of mass destruction have been found, the sense of urgency has been greatly reduced.”

\section{Risk of vaccination}

Almost all hospitals considered at least some aspect of the risks of vaccination: safety of the vaccine for vaccinees, the potential for transmission of vaccinia to patients or other close contacts of vaccinees, or the possible need to develop employee protocols to protect patients from exposure (e.g., furlough policies). Hospitals that chose to participate in the SPVP often decided that safety concerns could be addressed by educating employees, by carefully applying screening protocols, or by limiting the number of staff members deemed eligible for vaccination. An example provided by one respondent was:

"In asking for volunteers, we mainly asked for those that had been previously vaccinated because we were fairly confident that they would not experience a severe reaction." $\dagger$

In contrast, hospitals that decided not to participate in the SPVP had unresolved concerns about vaccine safety, or they determined that the risk of vaccination outweighed the risk of an outbreak:

"There was lack of good information about the safety of the vaccine."

"The main con was the potential for adverse effects from the vaccine, especially given that persons can be vaccinated within 72 hours of an actual outbreak and still be protected. We followed the physician motto of 'do no harm." "†

For many hospitals, the identification of myopericarditis as a potential side effect of smallpox vaccination was a particular deterrent and prompted some hospitals that initially chose to participate to suspend vaccination of their staff.

Exposure of immune-compromised patients, the elderly, and children to recent vaccinees was noted as a concern for about two-thirds of respondents overall. De- spite the ACIP/HICPAC guidelines, which indicate that staff could continue their normal working responsibilities if appropriate vaccine site care protocols were followed, many hospitals struggled over whether special policies needed to be developed for vaccinated employees. Some hospitals decided that the CDC protocols for vaccine site care were adequate, while others adopted stricter site care protocols or developed policies regarding eligibility for vaccination or reassignment:

"[Exposure of patients] was a big concern at first, but when we became more educated about the vaccine, it became less of a concern. We were confident in our site care policies and provided relevant information to patients of vaccinated employees." $\dagger$

"We decided that certain staff (e.g., OB nurses, staff in contact with chemotherapy patients) would not get vaccinated or would be reassigned." $\dagger$

"Vaccinees did not see patients during the period until their site healed. Nurses that worked with HIV or oncology patients were not asked to participate." $\dagger$

\section{Costs to the hospital}

Regardless of whether a hospital chose to participate in the SPVP, decision-makers were concerned about financial liability for employees injured as a result of vaccination who consequently became unable to work (i.e., workers' compensation) and the extent of liability for exposure of nonvaccinated individuals to vaccinia. While some hospitals made their decision before liability legislation was passed at the federal level, ${ }^{9}$ those that decided after federal liability provisions went into effect differed in their interpretation of the statute. Some hospitals felt that the legislation was inadequate, while others felt the provisions resolved their concerns. Some decision-makers believed that making vaccination voluntary relieved the hospital of liability:

"We felt it would be difficult to sue the institution because we made it very clear to staff that it was a voluntary program and also were very clear regarding the possible side effects." $\dagger$

Uncertainty about workers' compensation was a major concern and was addressed in various ways. Many hospitals determined that they were covered by workers' compensation policies already in place at the state level or, for some self-insured hospitals, at the individual hospital level. Some hospitals, or the corporations that owned them, agreed to compensate vaccinated workers who became ill or injured as a result of vaccination, at least until workers' compensation coverage kicked in. A few hospitals explicitly decided that employees would have to use 
sick or personal time off if it were needed, at least until the start of workers' compensation coverage.

About one-quarter of respondents indicated that costs related to lost work time or reassignment of staff influenced their decision-making process. Similar perspectives were shared by two hospitals, one that chose to participate in the SPVP and one that did not:

"The hospital is in a severe nursing shortage and it is very expensive to use agency nurses for any time off needed." $\dagger$

"We are small and worried that we would not be able to afford losing employees, either while getting the actual vaccine or in the event of adverse reactions."

\section{Stakeholder influences}

Hospitals were asked to discuss the influence on their decision of employees, the public, and outside parties such as government officials. Most hospital decisionmakers indicated that the influence of employees did not affect their decision-making process, because vaccination was voluntary for employees. However, hospital employee unions influenced some decision-makers against participating in the SPVP. Even though employees did not directly affect the decision-making process of many hospitals, the willingness of hospital employees to voluntarily be vaccinated determined the extent to which hospitals were able to form smallpox response teams.

Public perception of the hospital was important for about one-third of hospitals. These hospitals weighed the possibility that the public would perceive a decision to participate in the SPVP negatively-because smallpox cases would be directed to the hospital for treatment during an outbreak-or positively-because the hospital would be prepared to respond to an outbreak. As one hospital decision-maker indicated:

"We wanted the public to know we were preparing for this, but we didn't want to be labeled as 'the smallpox hospital' because that could mean lost revenue if the public avoided our hospital." $\dagger$

When explicitly asked, fewer than half of all hospitals indicated that outside parties such as local health departments or state governors influenced their decision-making process, although some interviewees did note external influences on their decision at other times during the interview. The most commonly mentioned outside parties influencing hospitals' decisions were local health departments, state health departments, the federal government, and other hospitals within the community or corporate network. When mentioned, the influence of local and state health departments was overwhelmingly positive. Several hospitals, regardless of whether they decided to participate in the SPVP, noted that their relationship with public health officials significantly improved during the decision-making process, and that they were better prepared in the future to work with public health agencies. Sample comments included:

"Local public health officials worked alongside us, and we developed a better relationship regarding the entire disaster planning program. It opened communication tremendously.” $\$$

"We have not collaborated with the health department in the past, and this new collaboration has created a good relationship." $\dagger$

In a few cases, interaction with public health officials had a negative influence on hospitals' decisions, as exemplified by the following statement from one respondent:

"The main reason we ended up not participating was due to the lack of preparedness and technical support from the local health department.” $\$$

Hospital decision-makers were split regarding the influence of the federal government. Positive comments focused on the CDC's helpfulness and the fact that there was a federal recommendation supporting vaccination. The feelings of hospitals citing negative experiences are reflected in the following comments:

\footnotetext{
"We were negatively influenced by the fact that the CDC did not take a firm stance on whether or not hospitals should participate.”

"We felt that the program was put together too quickly and that the state and the federal government were not able to adequately answer a lot of questions related to the program."†
}

Some hospital decision-makers were influenced by other hospitals in their community or corporate network. For example:

\footnotetext{
"The fact that other hospitals in our corporation chose not to [participate in the SPVP] made us more hesitant about doing so." $\dagger$

"We are part of a network of hospitals, and the rest of the hospitals chose to participate [in the SPVP], so we felt we should as well." $\dagger$
}

\section{Key Factors}

There were many competing issues for hospital decision-makers to consider when deciding whether to participate in the SPVP. The key factors for hospitals that chose to participate in the SPVP are listed in Table 2. The 
Table 2. Key Factor in Decision for Hospitals that Chose to Participate in the SMallpox Pre-Event Vaccination Program (SPVP)

\begin{tabular}{lc}
\hline Key factor & $\begin{array}{c}\text { Hospitals that chose to participate in SPVP } \\
(\mathrm{n}=88)\end{array}$ \\
\hline Responsibility to be prepared & $75 \%(66)$ \\
Option for employees to be vaccinated & $12 \%(11)$ \\
Risk of outbreak & $7 \%(6)$ \\
Other & $6 \%(5)$ \\
\hline
\end{tabular}

most commonly cited factor favoring a decision to participate in the SPVP was feeling a sense of duty or desire to be prepared in the event of an outbreak. This sense of responsibility typically extended to a hospital's patients, employees, and community, and in a few cases also to the national level:

"We have an obligation to provide health care to the community and to be prepared in the event of smallpox outbreak."†

"The staff felt strongly that we should be prepared as first responders and do the right thing as a patriotic duty." $†$

Key factors for hospitals that chose not to participate in the SPVP are listed in Table 3. The most commonly reported key factor was the perception that the risk of vaccination outweighed the risk of an outbreak of smallpox:

"The threat of a smallpox outbreak was not compelling enough to make administering the vaccine, with its risks and costs, worthwhile."

\section{DISCUSSION}

Hospitals took into consideration many complicated issues in deciding whether to participate in the SPVP. Nearly all hospitals in our study considered the risk of a smallpox outbreak, the risks associated with vaccination, and costs to the hospital in their decision; yet, decisionmakers with the same information came to very different conclusions about whether to participate in the SPVP. Most decision-makers in hospitals that chose to participate in the SPVP cited as most important the feeling that they have a civic duty to be prepared to respond in the event that a smallpox outbreak occurs. In contrast, the key factor for decision-makers in hospitals that chose not to participate in the SPVP was the perception that the risks of vaccination and the potential costs to the hospital outweighed the small risk of an outbreak occurring.

Given the occurrence of previously recognized ${ }^{10-12}$ but unexpected side effects (i.e., myopericarditis), ${ }^{13}$ the risks of vaccination may increasingly be a barrier to future smallpox pre-event vaccination efforts. Although for many hospitals in this study the decision to participate in the SPVP occurred before the cardiac risks were well documented, implementation of the program at the hospital level was often greatly affected. The number of workers actually vaccinated was often far fewer than the initial group of volunteers, largely because recognition of the cardiac risks made individuals ineligible for vaccination or caused them to reconsider their decision to be vaccinated.

As public health officials continue their efforts to evaluate and improve the infrastructure for responding to potential bioterrorist events, their ability to work successfully with hospitals in their community is critical. Even though state and local public health departments were

Table 3. Key Factor in Decision for Hospitals that Chose not to Participate in the Smallpox Pre-Event Vaccination Program (SPVP)

Key factor

Risk of vaccination greater than risk of outbreak

Logistical/cost issues

Unknowns surrounding the SPVP

Other
Hospitals that chose not to participate in SPVP $(\mathrm{n}=35)$

$57 \%(20)$

$23 \%(8)$

$9 \%(3)$

$11 \%(4)$ 
charged with assisting hospitals in forming response teams as part of the SPVP, fewer than half of the hospitals in our study reported that public health officials played an important role in their decision-making process. Almost all hospitals that did report that public health officials played a role in their decision felt their influence was positive. Taking advantage of the opportunity to be a positive influence on hospital decision-makers may help to address some of the barriers to SPVP participation identified in this study and re-energize smallpox preparedness efforts in the future.

Public health officials should ensure that hospital decision-makers are kept consistently well informed about the complex issues involved in implementation of a smallpox vaccination and response preparedness program. In addition, public health officials should stress the importance of hospitals as partners in bioterrorism preparedness efforts. Consistent messages and information should be conveyed across federal, state, and local levels of the public health infrastructure. Ongoing and open communication channels with hospitals and related stakeholders should be established and maintained. At the national level, federal health officials should partner with national groups that may influence hospital decision-makers, such as hospital associations or labor unions.

At the state and local levels, establishing good relationships with individual hospitals is paramount. In this study, hospitals that had positive experiences with public health officials were often engaged in collaborative efforts that brought all hospitals in a community or region together to discuss issues and receive the same information on topics such as vaccine safety and liability provisions. In future efforts, community or region-wide assessments of exposure risk and response capacity will inform the process of how future program efforts should be developed and improved.

Fostering discussion between hospitals and public health officials will likely allow hospital decision-makers to feel a greater stake in future vaccination program efforts and realize the importance to the public and their surrounding community of a unified response strategy. A more collaborative approach also may allow the needs and concerns of individual hospitals related to staff shortages, development of vaccination policies, and other issues to be taken into consideration.

There are several limitations to this study. First, the perspectives of the hospitals interviewed may not be representative of all acute care hospitals nationally. Second, the effect of local factors-both hospital-specific (e.g., location, size) and external (e.g., design of program and extent of outreach by local and state public health officials) - on hospital decision-making could not be assessed. These factors did vary widely across hospitals and states and should be addressed in future work. Also note that this study was intended to qualitatively assess hospitals' decision-making processes, not whether the hospitals that decided to participate ultimately had a successful program (i.e., vaccinated a team of individual health-care workers).

Nevertheless, this study provides an important insight into hospitals' decisions regarding whether to participate or not in the SPVP, including the range of factors considered and the role of outside parties. The results of this study suggest that further involvement of public health officials in establishing strong relationships with the hospitals in their communities - to help convey consistent messages across hospitals and to promote a communitywide strategy - may help foster broader participation of hospitals in bioterrorism preparedness efforts.

\section{ACKNOWLEDGMENT}

This study was funded by the Centers for Disease Control and Prevention through a cooperative agreement with the Association of Teachers of Preventive Medicine.

\section{REFERENCES}

1. Centers for Disease Control and Prevention. Recommendations for using smallpox vaccine in a pre-event vaccination program: Supplemental recommendations of the Advisory Committee on Immunization Practices (ACIP) and the Healthcare Infection Control Practices Advisory Committee (HICPAC). MMWR 2003;52(No. RR-7):1-16.

2. U.S. General Accounting Office (GAO). Smallpox vaccination: Implementation of national program faces challenges. Report to the Chairman, Committee on Governmental Affairs, U.S. Senate. April 2003. Report No. GAO-03-578. Available at: http://www.gao.gov/new.items/d03578.pdf. Accessed April 27, 2004.

3. Everett WW, Zaoutis TL, Halpern SD, Strom BL, Coffin SE. Preevent vaccination against smallpox: a survey of pediatric emergency health care providers. Pediatr Infect Dis J 2004;23:332-337.

4. Benin AL, Dembry L, Shapiro ED, Holmboe ES. Reasons physicians accepted or declined smallpox vaccine, February through April, 2003. J Gen Intern Med 2004;19:85-89.

5. Yih WK, Lieu TA, Rego VH, et al. Attitudes of healthcare workers in U.S. hospitals regarding smallpox vaccination. BMC Public Health 2003;3:20.

6. Everett WW, Coffin SE, Zaoutis T, Halpern SD, Strom BL. Smallpox vaccination: a national survey of emergency health care providers. Acad Emerg Med 2003;10:606-611.

7. Kwon N, Raven MC, Chiang WK, Moran GJ, Jui J, Carter RA, Goldfrank L; EMERGEncy ID Net Study Group. Emergency physicians' perspectives on smallpox vaccination. Acad Emerg Med 2003;10:599-605. 
8. Centers for Disease Control and Prevention. Smallpox Vaccination Program Status by State. Available at: http://www. cdc.gov/od/oc/media/spvaccin.htm. Last updated: October 31, 2004. Accessed December 3, 2004.

9. Smallpox Emergency Personnel Protection Act of 2003, Pub L No 108-20, 117 Stat 638 (April 30, 2003).

10. Dalgaard JB. Fatal myocarditis following smallpox vaccination. Am Heart J 1957;54:156-157.

11. Cangemi VF. Acute pericarditis after smallpox vaccination. N Engl J Med 1958;258:1257-1259.

12. Feery BJ. Adverse reactions after smallpox vaccination. Med J Aust 1977;2:180-183.
13. Centers for Disease Control and Prevention. Update: Adverse Events Following Civilian Smallpox VaccinationUnited States, 2003. MMWR 2004;53:106-107.

Address reprint requests to: Alex R. Kemper, $M D, M P H, M S$ 6 E08 300 North Ingalls Building Ann Arbor, MI 48109-0456

E-mail: kempera@med.umich.edu 